\title{
«КАСКАДНАЯ» СИСТЕМА АВТОМАТИЧЕСКОГО РЕГУЛИРОВАНИЯ С НЕСТАЦИОНАРНЫМ ОБЪЕКТОМ
}

"Cascade" system of automatic control of a non-stationary objects

Павлов А.И. ${ }^{1}$ (Pavlov A.I.)

${ }^{1}$ Одесская национальная академия пищевых технологий, г. Одесса

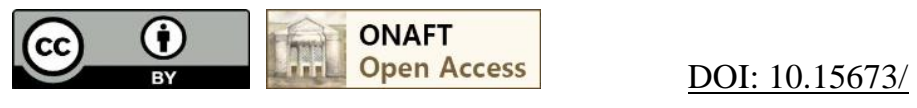

\begin{abstract}
Аннотация
Отмечается сложность настройки регуляторов каскадной системы автоматического регулирования. Сложность настройки особенно сильно возрастает, если объект регулирования нестационарный. Нестационарность проявляется в изменении величин коэффициентов дифференциальных уравнений объекта. Причина нестационарности заключается в том, что технологический агрегат объекта изнашивается («стареет»). Поэтому необходимы повторяющиеся процедуры настройки главного и подчиненного регуляторов. Это обстоятельство является одной из причин ограниченного использования каскадных систем регулирования. В статье утверждается, что при использовании линейных алгоритмов регулирования эта проблема не может быть устранена. Радикальным ее решением может быть использование в управляющей части системы искусственной нейронной сети. На примере регулирования температуры в теплице для выращивания овощей показана работоспособность этой концепции. В управляющей части системы использованы один нейрон и интегратор. Количество входных сигналов нейрона равно шести. Имитационное моделирование подтвердило, что качество регулирования не снижается при действии на объект больших координатных и параметрических возмущений. Подтверждено, что динамика системы регулирования сильно зависит от вида исполнительного механизма и его параметров.
\end{abstract}

\section{Abstract}

Notes the difficulty settings regulators cascade automatic control system. The difficulty settings are particularly aggressive, if the object of regulation of the non-stationary. The unsteadiness is manifested in the changing values of the coefficients of the differential equations of the object. The reason for the instability is that the technological unit of the object wears out («aging»). Therefore, the necessary duplicate procedure to configure the master and slave controllers. This fact is one reason for the limited use of cascade control systems. The article states that the use of linear control algorithms, this problem can not be resolved. Radical its solution can be use in the management part of the system of artificial neural network. In the control part of the system used one neuron and the integrator. The number of input neurons is equal to six. Simulation confirmed that the quality of regulation is not reduced by the action of the object coordinate large and parametric perturbations. Confirmed that the dynamics of the regulatory system strongly depends on the type of the actuator and its parameters.

Ключевые слова

Система регулирования, объект, модель, исполнительный механизм.

\section{Введение}

Каскадные системы автоматического регулирования (КСАР) применяются в тех случаях, когда объект состоит из двух последовательно соединенных участков. Если удается выявить в объекте наличие поддающейся измерению промежуточной координаты (выход первого участка объекта), то открывается практическая возможность построения КСАР, особенно эффективных в тех случаях, когда координатные возмущения действуют регулярно и сильно, в основном, именно на первый участок. 


\section{ПИТАННЯ ТЕОРЇ, МЕТОДИ ТА АЛГОРИТМИ ЕФЕКТИВНОГО АВТОМАТИЧНОГО УПРАВЛІННЯ ОБ'СКТАМИ ХІМІКО-ТЕХНОЛОГІЧНОГО ТИПУ}

До появления микропроцессорных средств управления КСАР в пищевой промышленности строились (в электрическом варианте их исполнения) обычно на основе приборов агрегатного комплекса «Контур». При этом в варианте использования аналогового сигнала управления в качестве регулирующих устройств использовались приборы К15; а в варианте использования импульсного сигнала управления в качестве главного регулятора использовался прибор К15, а ведомого - Р25.

Такие, и подобные им, КСАР требуют модернизации. Вместо аппаратных средств управления необходимо применять современные микропроцессорные регулирующие контроллеры. Однако только заменить аппаратные средства программируемыми без внесения существенных изменений в структуру управляющей части КСАР будет неправильно. И вот почему. Методика расчета оптимальных величин параметров обоих алгоритмов регуляторов, работающих совместно, отличается повышенной сложностью даже для периода пуско-наладочных работ по КСАР. А после введения в эксплуатацию, в результате износа объекта [3] и, как следствие, изменения численных величин его коэффициентов статики и динамики, требуется периодическая поднастройка управляющей подсистемы КСАР. Задача же эта несравненно более сложная, чем в случае обычной одноконтурной системы регулирования. Для выполнения такой работы на действующей КСАР требуется высококвалифицированный инженер-наладчик. Это является одной из причин не очень широкого использования КСАР.

Идеальным решением этой реальной проблемы было бы такое, чтобы однажды созданная и налаженная КСАР не требовала больше никогда выполнения подобного рода работ или, как минимум, на интервале времени между ремонтами технологического агрегата. В результате ремонтных работ параметры и характеристики объекта восстанавливаются полностью или, во всяком случае, воссоздаются близкими к первоначальным. Далее будет показано, что такая цель вполне достижима, при этом структура управляющей подсистемы не только не усложняется, а даже существенно упрощается: вместо тандема из двух регуляторов в классической КСАР вполне достаточным оказывается использование всего одного, но с иной структурой по сравнению с традиционной.

\section{Основная часть}

В качестве реального объекта, для которого целесообразно использование КСАР, была выбрана теплица для выращивания овощей. Основной регулируемой координатой для теплиц является температура внутри них. В [1] предложена КСАР температуры внутри помещения, при этом в качестве первого участка объекта регулирования выступает технологический узел (трубчатый теплообменник), обеспечивающий нагревание воздуха с последующей его подачей в производственное помещение. Вторым участком объекта является собственно производственное помещение, для которого (в зависимости от его объема) характерны большие величины запаздывания и инерционности, как по каналу управления, так и по каналу возмущения со стороны окружающей среды. Поскольку для теплиц типичен вариант рециркуляции воздуха, то для первого участка такого объекта регулирования не характерны возмущения ни по температуре воздуха на входе, ни по его расходу. Главным возмущением для него является возмущение по каналу управления - нестабильность давления водяного пара, используемого для нагревания воздуха. Это приводит к нестабильности температуры воздуха на выходе первого участка и, значит, в самой теплице. При таком варианте построения узла подогрева воздуха, для первого участка будут типичны только параметрические возмущения, обусловленные износом теплообменного аппарата.

Для второго участка объекта основным типичным возмущением является изменение температуры воздуха снаружи теплицы. Однако характерны (в той или иной мере) и параметрические возмущения.

Радикальным решением в отношении упрощения структуры управляющей подсистемы подобным объектом будет использование принципа простоты, известного как Бритва Оккама, а именно, отказ от использования двух регуляторов: ведущего и ведомого с их классическими линейными алгоритмами. Альтернативным и, как будет показано ниже, очень эффективным решением будет использование искусственной нейронной сети (ИНС) с той или иной ее структурой. Однако при выборе нейросети следует помнить, что с возрастанием ее размерности и количества обрабатываемых ею сигналов увеличивается результативность работы, но быстро растет и сложность обучения ИНС [2]. По этой причине использовался простейший ее вариант. Входные сигналы управляющей подсистемы: заданная величина температуры в теплице Yzdn, фактическая температура в ней Y(t), динамическая ошибка $\mathrm{E}(\mathrm{t})$, координатное возмущение первого участка fl(t), температура воздуха, подаваемого в теплицу (выходная координата первого участка) Y1(t), аналоговый сигнал управления $\mathrm{U}(\mathrm{t})$ и внешнее возмущение второго участка объекта $\mathrm{f} 2(\mathrm{t})$.

Работоспособность такой управляющей подсистемы исследовалась на имитационной модели, реализованной в среде MATLAB с использованием пакетов программ Simulink. Общий ее вид показан на рис. 1. 
ПИТАННЯ ТЕОРІї, МЕТОДИ ТА АЛГОРИТМИ ЕФЕКТИВНОГО АВТОМАТИЧНОГО УПРАВЛІННЯ ОБ'СКТАМИ ХІМІКО-ТЕХНОЛОГІЧНОГО ТИПУ

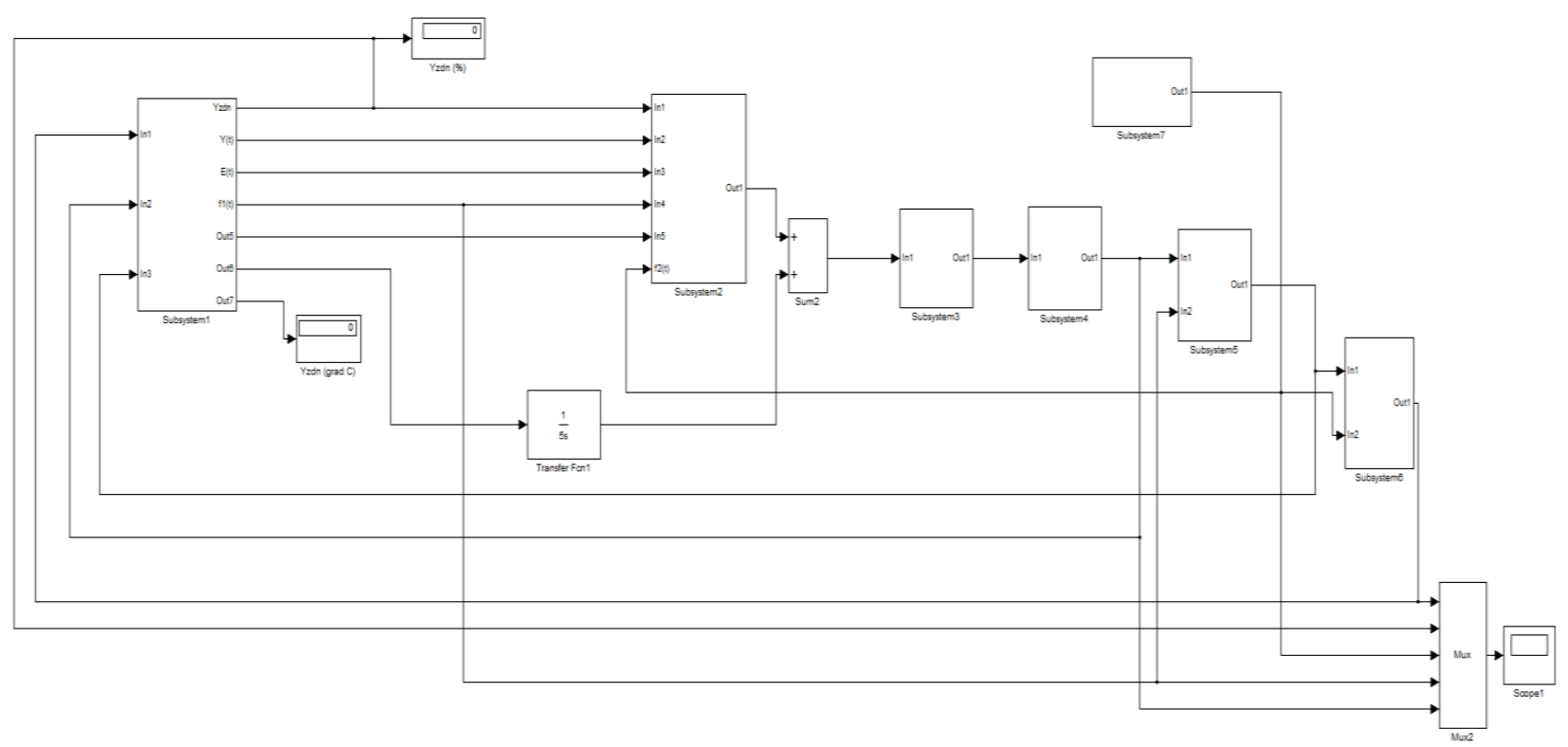

Рис. 1 - Структурная схема имитационной модели системы регулирования в среде MATLAB

Subsystem1 - подсистема формирования входных сигналов для нейронной сети;

Subsystem2 - нейронная структура; Subsystem3 - подсистема нормализации сигнала управления;

Subsystem4 - пакет из формализованных моделей исполнительных механизмов различных видов;

Subsystem5 - формализованная модель первого участка объекта регулирования;

Subsystem6 - формализованная модель второго участка объекта регулирования;

Subsystem7 - имитатор внешнего возмущения для второго участка модели объекта

Передаточная функция первого участка объекта в начале интервала времени расходования ресурса теплообменного аппарата [3]:

$$
\mathrm{W}_{1}(\mathrm{p})=\frac{1,6 \mathrm{e}^{-4 \mathrm{p}}}{(10 \mathrm{p}+1)^{2}}
$$

а в конце этого интервала времени

$$
\mathrm{W}_{1}(\mathrm{p})=\frac{0,8 \mathrm{e}^{-70 \mathrm{p}}}{(60 \mathrm{p}+1)^{2}}
$$

Приведенные передаточные функции отражают изменения параметров объекта, происходящие в течение времени между двумя ремонтами теплообменного аппарата.

Передаточная функция второго участка объекта:

$$
\mathrm{W}_{2}(\mathrm{p})=\frac{0,24 \mathrm{e}^{-300 \mathrm{p}}}{(150 \mathrm{p}+1)^{2}}
$$

соответствует величине помещения теплицы объемом в $3500 \mathrm{~m}^{3}$.

Существенная нестационарность свойственна и второму участку объекта - самой теплице по причине изменения во времени зеленой массы растений - от посадки рассады до сбора урожая. Это приводит к значительному снижению коэффициента передачи, увеличению инерционности и запаздывания. Поэтому при моделировании принято уменьшение величины (в сравнении с первоначальной) коэффициента передачи второго участка на $25 \%$, увеличение запаздывания на 100 секунд и инерционности на 75 секунд.

Таким образом, объект регулирования в целом следует отнести к числу сложных. Кроме того, допустимые отклонения температуры в теплице от задания довольно жесткие $\left( \pm 2{ }^{\circ} \mathrm{C}\right)$.

Пропускная характеристика регулирующего органа (парового клапана) соответствует равнопроцентной.

Как уже было отмечено [4], исполнительный механизм очень существенно влияет на динамику систем автоматического регулирования. Поэтому при имитационном моделировании использовались формализованные модели, как электрических исполнительных механизмов, так и пневматических мембранных, оснащенных 
позиционерами с различными алгоритмами. При использовании имитационных моделей электрических

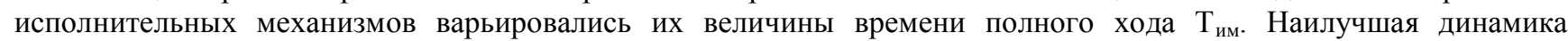
наблюдается при использовании в структуре имитационной модели варианта пневматического мембранного исполнительного механизма с пропорционально-дифференциальным алгоритмом его позиционера [4].

Непрерывное координатное возмущение по каналу управления первого участка модели объекта, характеризующее нестабильность давления пара, представлено суммой четырех синусоидальных сигналов с параметрами:

$$
\mathrm{A}_{1}=2, \mathrm{~F}_{1}=0,005 \mathrm{paд} / \mathrm{c} ; \mathrm{A}_{2}=4, \mathrm{~F}_{2}=0,001 \mathrm{paд} / \mathrm{c} ; \mathrm{A}_{3}=3, \mathrm{~F}_{3}=0,0015 \mathrm{paд} / \mathrm{c} ; \mathrm{A}_{4}=5, \mathrm{~F}_{4}=0,0001 \mathrm{paд} / \mathrm{c} .
$$

Закономерность суточных изменений наружной температуры (координатное возмущение для второго участка модели объекта) принята также синусоидальной с амплитудой $\mathrm{A}=20\left(8^{0} \mathrm{C}\right)$ и периодом колебаний 24 часа.

Характер и параметры имитируемых координатных возмущений были выбраны такими, чтобы регулирующий орган - при изменении коэффициента передачи первого участка объекта - перемещался во всем его диапазоне, то есть от 0 до 100 процентов.

Кроме этого имитационная модель системы регулирования была реализована с использованием ресурсов инструментальной системы программирования KONGRAF для промышленных контроллеров. Имитационная модель управляющей подсистемы, соответствующая Subsystem1, 2 и 3 на рис. 1, представлена на рис. 2.

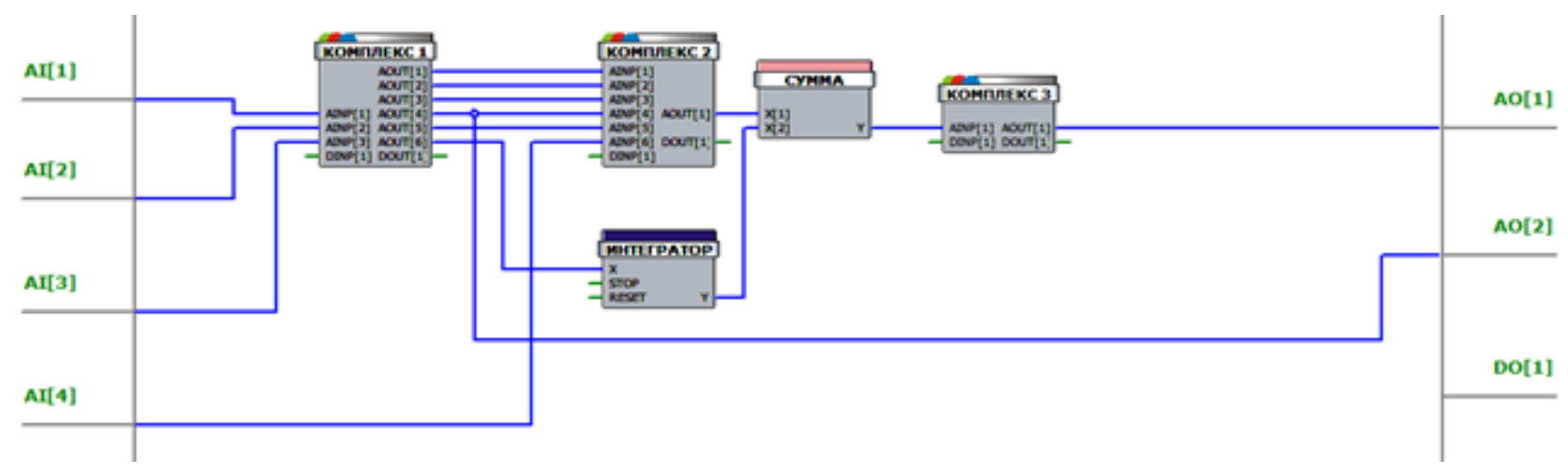

Рис. 2 - Структурная схема имитационной модели управляющей подсистемы в KONGRAF

Рассматривался вариант выращивания в теплице помидор. Оптимальная температура для них $20{ }^{0} \mathrm{C}$ с допустимыми длительными отклонениями $\pm 2{ }^{\circ} \mathrm{C}$.

Графические результаты моделирования представлены на рис. 3 и 4; показана динамика: температуры в теплице, координатных возмущений первого и второго участков объекта и управляющего воздействия. Все величины на графиках выражены в процентах, причем Yzdn = 50. Рис. 3 и 4 показывают, что перемещение затвора регулирующего органа при параметрах первого участка объекта, отображаемых формулой (1), соответствует (0...30) \% его полного диапазона, а при параметрах, отображаемых формулой (2), соответствует (70...100) \%. Таким образом, при моделировании использовался весь рабочий диапазон перемещения регулирующего органа с

присущими нелинейностями его расходной характеристики. В диапазоне же $(30 \ldots 70) \%$ хода регулирующего органа его расходная характеристика практически линейная. 


\section{ПИТАННЯ ТЕОРЇ̈, МЕТОДИ ТА АЛГОРИТМИ ЕФЕКТИВНОГО АВТОМАТИЧНОГО УПРАВЛІННЯ ОБ'ЄКТАМИ ХІМІКО-ТЕХНОЛОГІЧНОГО ТИПУ}

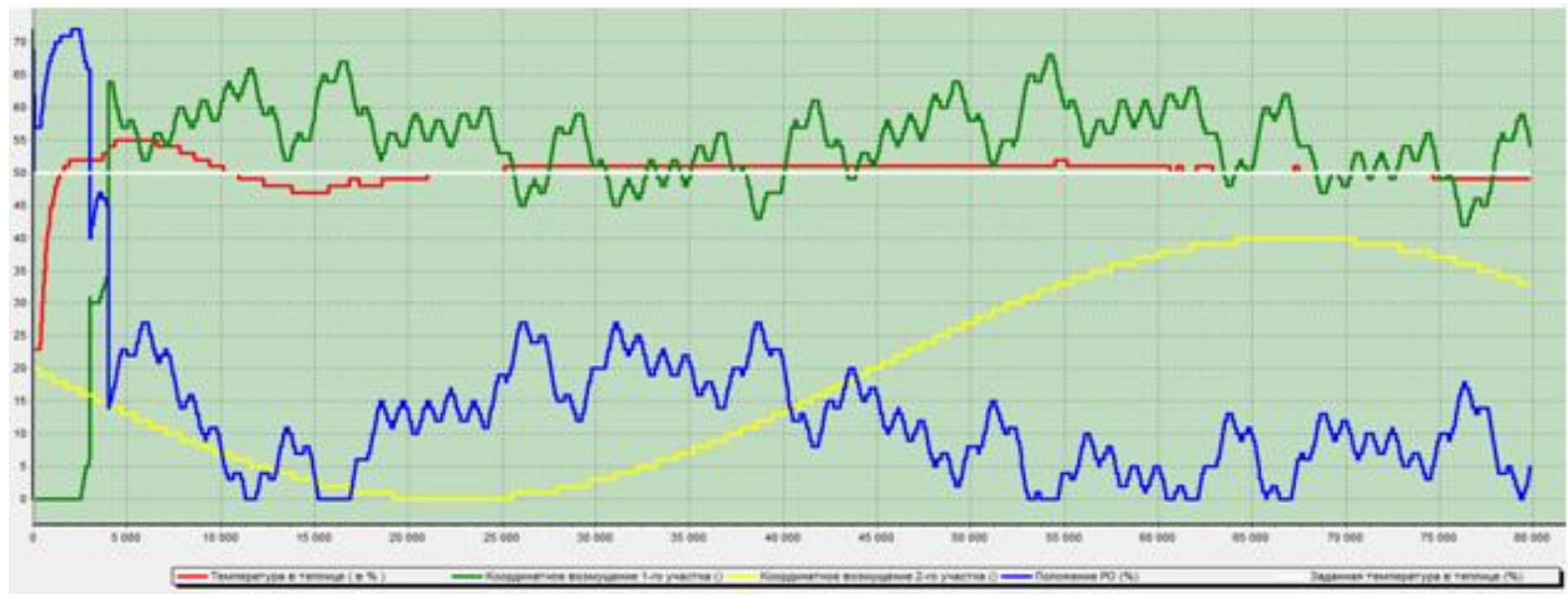

Рис. 3 - Переходные процессы при параметрах имитационной модели, соответствующих формуле (1)

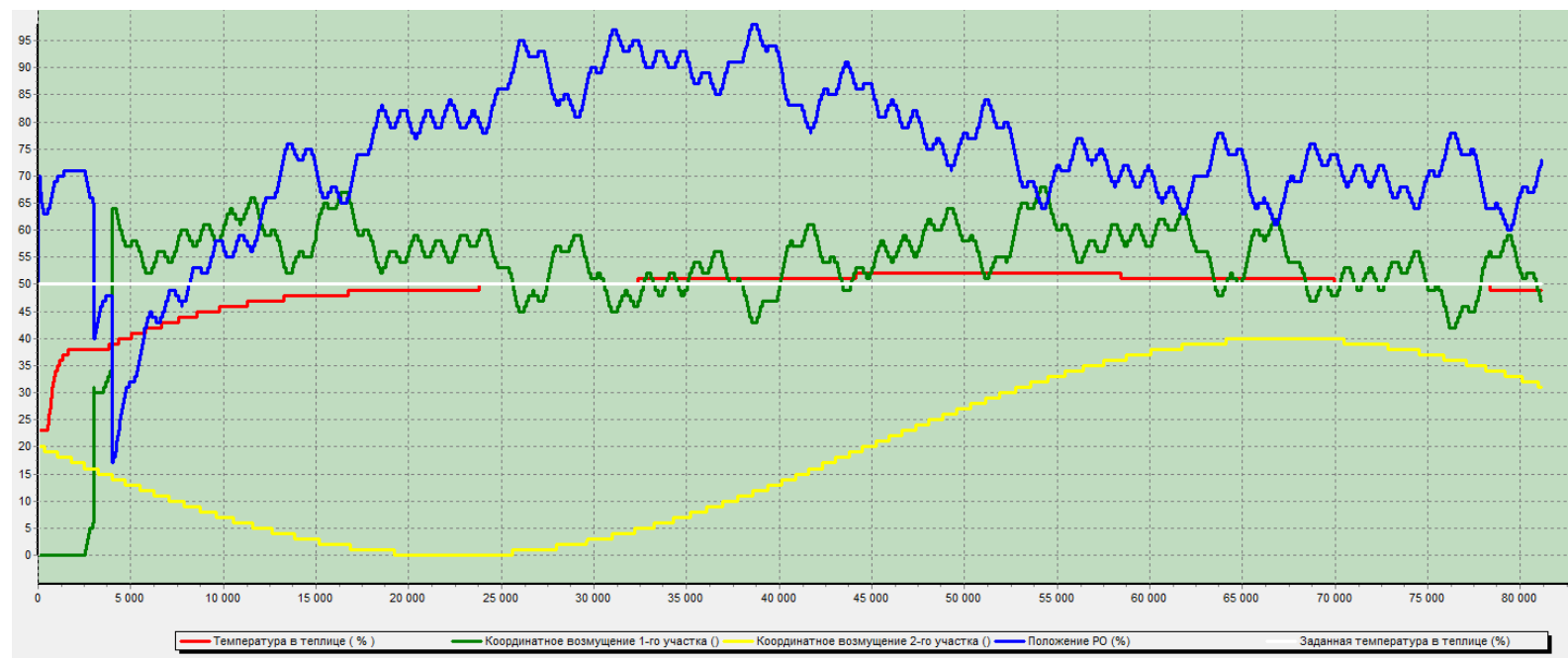

Рис. 4 - Переходные процессы при параметрах имитационной модели, соответствующих формуле (2)

\section{Выводы}

1. Предложенная структура системы автоматического регулирования, по совокупности особенностей и признаков, позволяет отнести ее к обыкновенным (несамонастраивающимся), комбинированным, нелинейным, нестационарным, робастным. Использование же в названии статьи термина «каскадная» имело своей целью привлечь внимание к актуальной проблеме модернизации реальных КСАР.

2. На расчетном (межремонтном) интервале времени расходования ресурса технологического аппарата (теплообменника первого участка объекта), имитационная модель системы регулирования показывает наличие адаптивных свойств как к большим координатным возмущениям первого участка объекта, так и к большим координатным возмущениям по каналу управления. Система регулирования хорошо демпфирует и большие координатные и параметрические возмущения второго участка модели объекта. Максимальная динамическая ошибка поддержания температуры в теплице не превышает $0,75{ }^{0} \mathrm{C}$. Более того, по мере износа теплообменника (снижения коэффициента передачи первого участка объекта, увеличения его инерционности и запаздывания), качество регулирования не только не снижается, а напротив - возрастает! Динамическая ошибка снижается до 0,5 ${ }^{0} \mathrm{C}$, при том, что в процессе моделирования никакие изменения в управляющей подсистеме, и вцелом в модели, не производились.

3. Имитационное моделирование показало сильное влияние на динамику КСАР как вида исполнительного механизма, так и его характеристик: в вариантах использования электрических исполнительных механизмов динамическая ошибка значительно больше, достигая $1,5{ }^{0} \mathrm{C}$ (при оптимальных величинах постоянных времени $\mathrm{T}_{\text {им}}$ ), однако не выходит за пределы регламентной зоны. Следует отметить также, что принятые при 


\section{ПИТАННЯ ТЕОРЇ̈, МЕТОДИ ТА АЛГОРИТМИ ЕФЕКТИВНОГО АВТОМАТИЧНОГО УПРАВЛІННЯ ОБ'ЄКТАМИ ХІМІКО-ТЕХНОЛОГІЧНОГО ТИПУ}

моделировании величины координатных и параметрических возмущающих воздействий обусловили перемещение затвора регулирующего органа во всем его диапазоне и, значит, нелинейность его пропускной характеристики отразилась на динамике модели системы регулирования в полной мере.

\section{Литература}

1. Рымкевич А. А. Управление системами кондиционирования воздуха [Текст] / А. А. Рымкевич, М. Б. Халамайзер. - М. : Машиностроение, 1977. - 274 с.

2. Павлов А. И. Технология проектирования нейронных регуляторов [Текст] / А. И. Павлов // Автоматизация технологических и бизнес-процессов. - Одесса : ОНАПТ, 2010. - № 4. - С. 15-20.

3. Воинова С. А. Управление технологическими объектами и техническая геронтология [Текст] / С. А. Воинова // Автоматизация технологических и бизнес-процессов. - Одесса : ОНАПТ, 2011. - № 7, 8. - С. 20-23.

4. Павлов А. И. Виртуальный стенд для определения оптимального по быстродействию исполнительного устройства [Текст] / А. И. Павлов // Автоматизация технологических и бизнес-процессов. - Одесса : ОНАПТ, 2014. - № 4. - Т. 6. - С. 130-137.

\section{References}

1. Rymkevich A. A. Upravleniye sistemami konditsionirovaniya vozdukha [Tekst] / A. A. Rymkevich, M. B. Khalamayzer. - M. : Mashinostroyeniye, 1977. - 274 str.

2. Pavlov A. I. Tekhnologiya proektirovaniya neyronnykh regulyatorov [Tekst] / A. I. Pavlov // Avtomatizatsiya tekhnologicheskikh i biznes-protsessov. - Odessa : ONAPT, 2010. - № 4. - S. 15-20.

3. Voinova S. A. Upravleniye tekhnologicheskimi ob'ektami i tekhnicheskaya gerontologiya [Tekst] / S. A. Voinova // Avtomatizatsiya tekhnologicheskikh i biznes-protsessov. - Odessa: ONAPT, 2011. - № 7, 8. - S. 20-23.

4. Pavlov A. I. Virtual'nyy stend dlya opredeleniya optimal'nogo po bystrodeystviyu ispolnitel'nogo ustroystva [Tekst] / A. I. Pavlov // Avtomatizatsiya tekhnologicheskikh i biznes-protsessov. - Odessa : ONAPT, 2014. - № 4. - T. 6. - S. 130-137.

\section{Е НОВОСТИ АВТОМАТИЗАЦИИ}

\section{7-го сентября 2015 года состоялся финал конкурса Сатоzzі - на лучиую разработку промышленного робототехнического комплекса.}

По условию конкурса команда - победитель получает финансирование компании Camozzi для сборки своего робота физически. На открытии конкурса изначально анонсировали свое намерение и были допущены к участию студенты трех факультетов академии: факультета автоматизации, электромеханики, компьютерных систем и управления (АЕКСиУ), факультета технологического оборудования, упаковки и технического дизайна (ТОУиТД) и факультета информационных технологий и кибербезопасности (ИТиК). К старту конкурса, который состоялся в мае этого же года, участникам было предложено 5 задач, к финалу добрались только 3 команды, да и то не полным составом.

Итог финала конкурса - все три проекта одобрены для физической реализации, и соответственно для всех трех проектов компания Camozzi предоставляют необходимое современное оборудование для сборки роботов воплоти. Подробный фото-отчет доступен в альбоме группы лаборатории в контакте (vk.com/onapt_laboratory) и на официальном сайте лаборатории

http://robot.onaft.edu.ua

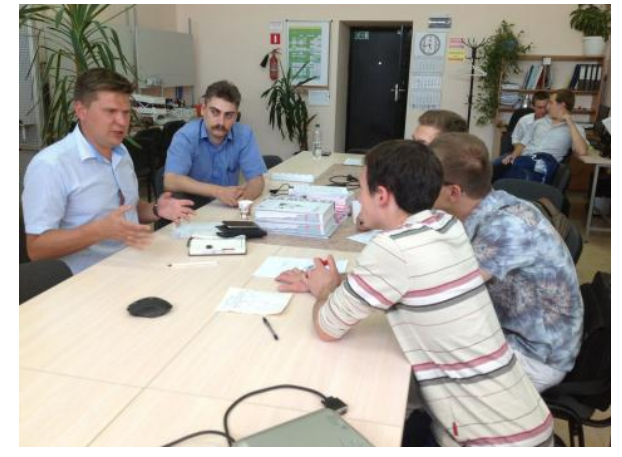

